\title{
Melanin Nanoparticles as an Alternative to Natural Melanin in Retinal Pigment Epithelium Cells and Their Therapeutic Effects against Age-Related Macular Degeneration
}

Yong-Su Kwon ${ }^{1}$, Min Zheng ${ }^{1}$, Alice Yang Zhang ${ }^{1}$, and Zongchao Han ${ }^{1,2, *}$

${ }^{1}$ Department of Ophthalmology, The University of North Carolina at Chapel Hill, Chapel Hill, NC 27599, USA

${ }^{2}$ Division of Pharmacoengineering \& Molecular Pharmaceutics, Eshelman School of Pharmacy, the University of North Carolina at Chapel Hill, Chapel Hill, NC 27599, USA

*Correspondence to: Zongchao Han, zongchao@med.unc.edu

Key Words: age-related macular degeneration, melanin nanoparticles, natural antioxidant, reactive oxygen species, intracellular trafficking

\begin{abstract}
Melanin is a natural pigment that is widely distributed in many parts of the human body, such as the skin and retinal pigment epithelium (RPE) in eyes. In contrast to skin melanin, which is being constantly synthesized by the epidermal melanocytes, melanin in the RPE does not regenerate. Melanin is known to function as a potential radical scavenger and photoprotective agent. However, the protective effects of melanin against oxidative stress decline with increasing age. This phenomenon has been significantly correlated with the pathogenesis of age-related macular degeneration (AMD). To increase the potential antioxidant and photoprotective characteristics of the melanin, we design new strategy by replenishment of melanin using PEGylated-synthetic melanin nanoparticles (MNPs) in the RPE for the treatment of AMD. We perform experiments using AMD-like cellular and mouse models and demonstrate that MNPs are safe, biocompatible, and selectively target reactive oxygen species (ROS) with powerful antioxidant properties. MNPs can traffic and accumulate in the RPE and are exclusively located in cytosol but not the nucleus and mitochondria of the cells for up to three months after a single-dose intravitreal (IVT) injection. Our findings demonstrate, for the first time, that MNPs are able to substitute for natural melanin in the RPE of eyes and suggest the potential efficacy of MNPs as a natural radical scavenger against oxidative stress in ROS-related diseases, such as AMD.
\end{abstract}




\section{Graphical Abstract}

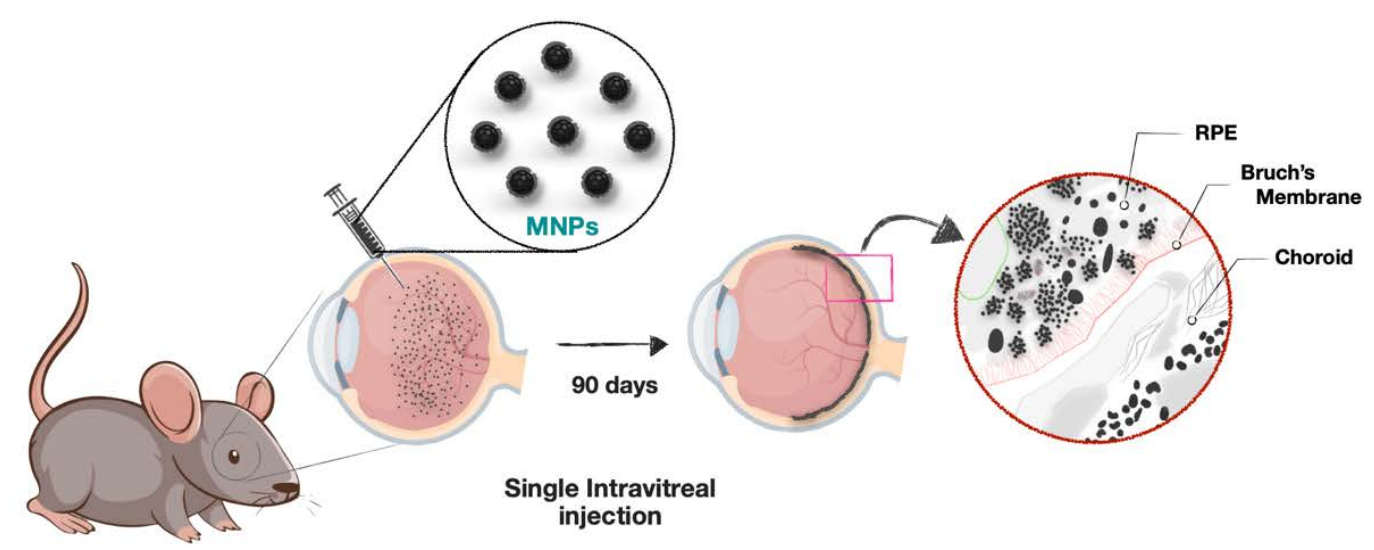

Wild type mouse (C57BL/5J)

\section{Introduction}

Age-related macular degeneration (AMD) is a leading cause of blindness in elderly patients with a well-known public health impact, which is classified into two main pathological condition: the non-exudative (i.e., dry) and exudative (i.e., wet or neovascular) forms. ${ }^{1}$ Despite significant advances in therapeutic options, the AMD patient population has continued to grow. ${ }^{2}$ As current treatments for dry AMD are ineffective, there is a major need for therapies for dry AMD. Although treatments for wet AMD are effective, they are costly and are associated with a heavy burden of health care, and therefore there is a major need for improvements in treatments for wet AMD. ${ }^{3}$ The retina is one of the highest oxygen consuming tissues in the human body. The increasing production of reactive oxygen species (ROS) in the retina is affected by continual exposure to light, high concentrations of polyunsaturated fatty acids, and intensive oxygen metabolism. ${ }^{4}$ The overproduction of ROS by chronic oxidative stress can exceed the antioxidant capability of the retina and lead to damage of carbohydrates, membrane lipids, and proteins and nucleic acids in the retina, which are significant contributors to the development of AMD. ${ }^{5-6}$ Increasing evidence shows that oxidative stress has played a decisive role in the progression of both wet and dry forms of AMD, representing a potential therapeutic target for AMD. ${ }^{7-11}$ Hence, a long-term sustained delivery of antioxidants for neutralizing oxidative stress may lead to a novel treatment targeted to preserve the retinal pigment epithelium (RPE) and photoreceptor cells in AMD. 
Melanin is a well-known natural antioxidant, which plays a key role in protecting retinal cells from light-generated free radicals. ${ }^{12}$ Melanin is synthesized by L-DOPA, a precursor for the formation of melanin, during embryogenesis in RPE cells. However, it does not regenerate on adult RPE cells. ${ }^{13-14}$ The antioxidative activity of melanin in the RPE cells declines with increasing age. ${ }^{15-16}$ Thus, maintaining the level of healthy melanin in RPE cells is very important because RPE cells are vulnerable to detrimental effects from aged melanin.

Very convincing evidence and clinically relevant data now support that nanomaterials have shown great promise as potential new tools in therapeutic approaches for ocular diseases. ${ }^{17-18}$ Recently, dopamine-derived synthetic melanin nanoparticles have been suggested as a novel nanoantioxidant in biomedical applications with its highly biological relevance to natural melanin. ${ }^{19}$ We investigate the trafficking pathway and the final destination of PEGylated-synthetic melanin nanoparticles (MNPs) in biological systems and determine whether MNPs can be an alternative as natural melanin of RPE cells in vitro and in vivo. Our results provide strong evidence for their roles in attenuating pathological damages in AMD models. To the best of our knowledge, the melanin-based nanoantioxidants have not been studied in ophthalmic application yet, and there is still plenty of opportunity for discovery. We develop a safer and more effective antioxidant that can potentially achieve long-term effect through a single-dose administration to relieve pathological damages for AMD.

\section{Results}

\subsection{Synthesis and characterization of melanin nanoparticles}

The synthetic melanin nanoparticles (Bare-MNPs) were produced by oxidative polymerization of dopamine hydrochloride in the presence of sodium hydroxide as previously reported with slight modifications. ${ }^{20}$ The generation of Bare-MNPs were observed by the color of the solution that immediately turned to pale yellow and gradually became dark brown. Subsequently, thiolterminated methoxy-polyethylene glycol (mPEG-SH) was introduced on the surface of Bare-MNP by linkage via Michael addition reaction ${ }^{21}$ to improve the physiological stability of Bare-MNPs (Figure 1a). After the reaction, the prepared MNPs were observed to have an average diameter of $\sim 80 \mathrm{~nm}$ with uniform nanospheres from transmission electron microscopy (TEM), which did not show any morphological difference after surface modification (Figure 1b, See Figure S1 in the Supporting Information). Hydrodynamic size was measured by dynamic light scattering analysis 
(DLS) to determine the size distribution of nanoparticles. The DLS size of MNPs was $100 \mathrm{~nm}$ and slightly increased to $\sim 4.5 \mathrm{~nm}$ or less after surface modification compared to corresponding Bare-MNPs (Figure 1c). The zeta-potential analysis of these nanoparticles observed negative values, which was attributed to the multiple catechol groups on the melanin surface. Moreover, the resulting MNPs showed highly negative potential of - $48.2 \mathrm{mV}$ (Figure 1d) due to the result of formation of a methoxy PEG layer with slightly negative charge on their surface, which was confirmed by Fourier transform infrared (FT-IR) spectra analysis (Figure 1e). The characteristic spectra peaks of MNPs showed the main absorption at $2880 \mathrm{~cm}^{-1}$ (the alkyl C-H stretching vibration) and $1110 \mathrm{~cm}^{-1}$ (the $\mathrm{C}-\mathrm{O}-\mathrm{C}$ stretching vibration) that were assigned to the PEG molecules. In terms of dispersion stability, MNPs revealed excellent dispersion ability in monkey vitreous for three months without particles aggregation (Figure 1f, Figure S2 supporting information) as well as the high colloidal stability was exhibited in saline, culture media, and deionized water for a month (Figure S3 supporting information). These results validate that longterm stability of MNPs in biological media is suitable for biological applications.

\subsection{Scavenging activity and intracellular uptake behavior of MNPs}

Based on the distinct antioxidant properties of melanin in the biological system, we assessed the potential of MNPs to act as robust ROS scavengers. Here, we performed a 2, 2-diphenyl-1picrylhydrazyl (DPPH) assay, which is widely used to predict the free radical scavenging capacity. ${ }^{22}$ DPPH produces purple color in methanol solution and fades to yellow color in the presence of antioxidants. We evaluated the capacity by monitoring the absorbance change at 516 $\mathrm{nm}$. As shown in Figure 2a, MNPs exhibited excellent scavenging activity of $\approx 82 \pm 2.5 \%$ for free radicals at the dose level of $100 \mu \mathrm{g} \mathrm{mL}^{-1}$ compared with $0 \mu \mathrm{g} \mathrm{mL}^{-1}$ (PBS control). Moreover, the MNPs-mediated scavenging activity was verified through the concentration-dependent suppression behavior toward free radicals within our experimental dose range of $0-100 \mu \mathrm{g} \mathrm{mL}$ 1. We confirmed the highly sensitive antioxidant capacity of MNPs to scavenge of free radicals. The result suggests that it shows free radical scavenging activity by their electron donating or accepting ability of MNPs. ${ }^{23}$

\subsection{MNPs-mediated protection on $\mathrm{H}_{2} \mathrm{O}_{2}$-induced oxidative stress cells.}

An imbalance between the production of ROS and the antioxidant defenses is implicated in persistent oxidative stress in RPE cells. Excessive accumulation of ROS leads to nonspecific 
inflammation and has been shown to be a potential factor in the pathogenesis of AMD. ${ }^{6,10-11}$ To determine scavenging ability of MNPs, we evaluated the amount of intracellular ROS levels in ARPE19 cells by DCF-DA fluorescence assay, which is used widely as a nonspecific indicator of ROS levels in cultured cells. We measured the intracellular ROS levels with $0.576 \mu \mathrm{M}$ hydrogen peroxide $\left(\mathrm{H}_{2} \mathrm{O}_{2}\right)$ that is sufficient to increase the significant amount of DCF fluorescence in ARPE 19 cells. ${ }^{18} \mathrm{H}_{2} \mathrm{O}_{2}$-induced ROS production in ARPE19 cells was significantly suppressed by MNPs in comparison to untreated $(0 \mu \mathrm{g})$ and DCF-DA (reagent only)-treated controls with the increase in the concentration of MNPs (Figure 2b). Intracellular ROS generation increased by about 7.5fold upon addition of $\mathrm{H}_{2} \mathrm{O}_{2}$, whereas it clearly decreased upon treatment with MNPs $(2-10 \mu \mathrm{g}$, ${ }^{*} \mathrm{p}<0.05, * * \mathrm{P}<0.01$, and $\left.* * * \mathrm{P}<0.001\right)$. We further examined whether MNPs affected the fluorescence of the DCF-DA in absence of $\mathrm{H}_{2} \mathrm{O}_{2}$. The separated experiment showed that there were no interactions between DCF-DA and MNPs (2 - $10 \mu \mathrm{g}$, ns), which remained comparable to the fluorescence of DCF-DA (reagent only)-treated cells (Figure 2c). These results revealed that MNPs did not create any intracellular ROS. For all concentrations of MNPs, no obvious cytotoxicity was revealed under the experimental conditions, as observed through water-soluble tetrazolium salt (WST-1) assay (Figure S2, Supporting Information). Additionally, ROS accumulation in the ARPE19 cells via administration of $\mathrm{H}_{2} \mathrm{O}_{2}(0.576 \mathrm{mM})$ were observed using MitoSox, a cell-permeable indicator that recognizes mitochondria-specific ROS. We confirmed that MNPs can be efficiently taken up into the ROS-induced ARPE19 cells (Figure S5 supporting information), and the exceeded ROS was significantly inhibited in the presence of MNPs (Figure 2d).

\subsection{Visualization of MNPs in ocular tissues.}

To evaluate whether MNPs reach their targets after administration, we performed TEM to visualize where the nanoparticles go both in vitro in ARPE19 cells and in vivo within the eye via IVT injection. The physiological levels of ROS in mitochondria play an essential role in the redox state and signaling in normal cellular metabolism. However, disruption of ROS balance in mitochondria can lead to the development of oxidative stress by excessive production of ROS that overflow to the cytosol from the mitochondria. ${ }^{24}$ To visualize the uptake pathway of MNPs by the ARPE19 cells, we used a Bio-TEM analysis with an epoxy resin embedding method. Compared with un-treated group, we found that MNPs were exclusively located in cytosol after endocytic 
uptake and not in the nucleus and mitochondria of the transduced cells (Figure 2e), which was more prominent at various magnifications (Figure S6 supporting information). To investigate whether MNPs accumulation and distribution change in retinal cells, we injected $1 \mathrm{uL}$ of MNPs (10 $\left.\mu \mathrm{g} \mathrm{uL}^{-1}\right)$ via single-dose IVT injection and evaluated the trafficking of the nanoparticles at 3 , 15, 30 and 90 days post-injection in wild type mice. Interestingly, We found that MNPs were exclusively accumulated in the RPE cells (Figure 3b), and its accumulation was gathering over time and they were not able to cross the Bruch's (Br) membrane (Figure 3c-e). Similar to the in vitro trafficking study as described in Figure 2e, they were not found in the melanosomes, and mitochondria in the RPE (Figure S7 supporting information), indicating that MNPs are capable of targeting free radicals that overflew to the cytosol.

\subsection{Neovascularization inhibition in laser-induced CNV mouse model}

To demonstrate that the antioxidants of MNPs confer a benefit in AMD, we administered IVT injection of MNPs on a laser-induced CNV in C57BL/6J mice. The fundus fluorescein angiography (FFA) and optical coherence tomography (OCT) showed the presence of laser injuries immediately after the laser photocoagulation (top panels in Figure 4a, b and c). After FFA and OCT analysis of the CNV lesion (i.e., before injection), we performed an IVT injection with $1 \mathrm{uL}$ of saline, aflibercept (40 $\mu \mathrm{g} \mu \mathrm{L}^{-1}$ ), and MNPs (1 $\mu \mathrm{g} \mu \mathrm{L}^{-1}$ ) to each laser-treated mouse eye, respectively. The FFA images showed that the MNPs-treated group clearly revealed a reduced vascular leakage from CNV lesions compared to the saline-treated group at 14 days after laser photocoagulation (bottom panels in Figure 4a and c). The OCT confirmed the presence of the rupture of $\mathrm{Br}$ membrane immediately after the laser photocoagulation (indicated by arrows in Figure 4a, b and c). We also followed up retinal pathology with 2D cross-sectional OCT scans and confirmed presence of the rupture of $\mathrm{Br}$ membrane immediately after laser photocoagulation (indicated by arrow in Figure 4a, b and c). The OCT analyses showed that the thickness of laserinduced CNV injuries was significantly reduced in the MNPs-treated group compared to the salinetreated group at 14 days post-injection (Figure 4a and c). Similarily, the therapeutic effect of aflibercept was significantly higher than that of the saline-treated group. However, we observed that MNPs have more potent antiangiogenic activity than that of aflibercept (tradename EYLEA), an FDA-approved drug which targets VEGF. A statistically significant difference was found between the two groups $(\mathrm{p}<0.01)$ (Figure $4 \mathrm{~b}$ and $\mathrm{c}$ ). 
The typical morphology of vascular area of laser-induced CNV lesions can be well quantitatively analyzed by choroidal flat mounts. To quantify the degree of CNV lesions, we prepared RPE/choroid/scleral flat mount specimens at 14 days post laser photocoagulation by staining with Alexa Fluor 488-conjugated isolectin-IB4 (IB4). IB4 is a commonly used endothelial cell specific markers. The areas of neovascularization were assessed using an Axiocam MR 5 camera on an Axio Observer.D1 inverted microscope (Carl Zeiss, Norway). We observed that the size of IB4labeled CNV outgrowths was markedly reduced in the MNPs-treated group compared to that of the saline and aflibercept-treated groups (Figure 5a). The total area of CNV associated with each burn was measured per eye using Image J software (Figure 5b). The average size (in $\mu \mathrm{m}^{2}$ ) of CNV lesion per eye significantly decreased in the MNPs-treated group compared with saline- and aflibercept-treated groups ( $\mathrm{p}<0.001)$. Thus, our results suggest that CNV lesions can be suppressed by antioxidant activity of MNPs.

\subsection{Direct IVT delivery of MNPs is safe and has no toxicity to ocular tissues}

It investigate whether IVT delivery of MNPs result in any toxicity or morphlogical changes in the eye, we conducted histologic analyses of the retinas. The outer nuclear layer (ONL) thickness serves as an important biomarker to monitor retinal pathological changes for visual function. ${ }^{25}$ Changes of ONL thickness are connected to toxicity to the ocular tissues. The measurements of ONL thickness in superior and inferior hemispheres was performed to evaluate the thickness changes after injection $1 \mu \mathrm{L}$ of saline and MNPs $\left(1 \mu \mathrm{g} \mu \mathrm{L}^{-1}\right)$ inside the vitreous space without laser photocoagulation. Mice were sacrificed at 5 days after injection and retinal cross-sections were prepared for histological analyses. Compared with saline-treated group, we did not find any morphological changes nor the effect on the retinal degeneration or any signs of inflammatory changes in the ocular tissues in the MNPs-treated group (Figure 5c, d, and e).

\section{Discussion}

In this study, we investigated the intracellular trafficking pathway and therapeutic capability of MNPs as a novel nanoantioxidant for AMD therapy. There is increasing evidence that suggests dysfunction of melanin contributes to the pathogenesis of AMD. ${ }^{26}$ A study by Weiter et al. shows that there are about 2-fold higher density of melanin in the human macula area compared to that of the paramacular area in the RPE. ${ }^{27}$ It is well known that Caucasian people are more likely to develop AMD than African American people. ${ }^{2}$ This phenomenon may have been in part attributed 
by the different levels of melanin between the races. Studies have shown that compared with pigmented $\mathrm{Abca} 4^{-/-}$mice, early onset of oxidative stress and lysosomal impairment within RPE were found in albino Abca4 $4^{-/-}$mouse model of Stargardt macular dystrophy. ${ }^{12,28}$ Indeed, a similar study has been conducted in the mid-70s using congenic strains of rats which defer in melanin pigmentation. ${ }^{29}$ The post-mitotic RPE cells are derived from the neuroectoderm (neural tube) - the embryonic precursor, which is known to develop into the central nervous system. The melanosomes in the RPE are synthesized during the prenatal period and shows very little, if any, turnover after birth. An age-related decline occurs in either reduction of quantity or quality of melanin in the adult RPE. Conversely, lipofuscin (A2E), a major component of a toxic product known as "age pigment", is found in AMD patients and is actively deposited on melanin granules throughout life. ${ }^{30}$ This phenomenon correlates with the stages of progression of lipid peroxidation and protein oxidation of cellular components in AMD pathology. Therefore, replenishment of melanin in the RPE in AMD patients could suppress retinal susceptibility to oxidative damage and represents a novel approach.

We prepared bioinspired Bare-MNPs using a simple coordination and self-assembly strategy, followed by PEGylation to create MNPs (approximately $80 \mathrm{~nm}$ ) exhibiting excellent solution stability and biocompatibility with antioxidative activity against oxidative stress. Solution stability is a very important aspect in the evaluation for future experiments in clinical investigation. MNPs exhibited long-term solution stability in the monkey vitreous (for three months, last time checked) as well as the aqueous solution (for 12 months, data not shown) without particle aggregation. MNPs contain abundant hydroquinone residues that can serve as a trap for potentially harmful free radicals, which can protect cell damage from oxidative stress. ${ }^{31}$ We observed that MNPs can work as an efficient free radical scavenger as revealed by DPPH assay, which has a function similar to the eumelanin in the RPE, to protect ARPE19 cells against harmful oxidative stress without affecting cell viability.

Nanoparticles could easily pass through the biological barriers and enter the cells via pino- or macropinocytosis. ${ }^{32}$ We found that MNPs localized predominantly in the cytosol and do not enter the mitochondria or nucleus of transduced cells as described Figure 2e. Given that mitochondria act as a source of ROS as redox signaling and that physiological levels of ROS are essential for normal cellular metabolism, we presume that it is much safer to access the intracellular ROS 
through scavenging than that of directly going to mitochondria because it will not target the physiological levels of ROS in mitochondria that are essential for normal cellular metabolism but will only target the "bad ROS" that overflow to the cytosol. This phenomenon was further confirmed by in vivo trafficking study via a single IVT injection in ocular tissues. One of the most noticeable advantages of MNPs is that the nanoparticles prefer to accumulate in the RPE layer and do not pass through the Br membrane after a single-dose IVT injection for up to three months (last time checked). MNPs were evenly dispersed in the RPE layer similar to the in vitro trafficking studies we found that the nanoparticles do not enter nucleus or mitochondria in the RPE (Figure 2e, Figure S7 supporting information). The distribution behavior of the MNPs (i.e., remain in the cytosol and do not enter into the mitochondria or nucleus), is critical as this will allow cells to maintain their normal cellular metabolism and to keep their nucleus genetic material integrity without being influenced by the nanoparticles. These findings suggest that MNPs could function as a key factor to modulate the activity of melanin in the RPE without inducing any toxicity, providing insights into future directions using MNPs as a novel approach to overcome dysfunction of melanin. After confirming the trafficking pathway of MNPs in ocular tissues, we investigated the therapeutic effect of MNPs in the laser-induced CNV mouse model. Our results showed that MNPs could reduce the pathological injuries in CNV and are even superior to aflibercept, an FDA approved prescription medication, in the CNV mouse model, indicating that MNPs play an important role as a robust antioxidant against pathological ROS (Figure 4). Compared to major anti-VEGF agents (aflibercept, ranibizumab, and bevacizumab) that may subject to issues of short half-life (7-10 days) and the effects of antibody uptake (e.g., diffusivity, antigen density, permeability, and systemic clearance), ${ }^{33-34}$ MNPs-based therapeutic system has improved bioavailability, increase resistance to cellular enzymatic digestion, and extend retention period, which has considerable key advantages. The fact that the MNPs-treated retinas are structurally similar to that of WT control, as indicated by the histological analysis (Figure 5d, e), made it a superior antioxidant and as a therapeutic tool to relieve pathological damage for AMD.

\section{Conclusion}

In conclusion, we demonstrated the efficacy of the bioinspired melanin nanoparticles as a natural antioxidant defense platform for AMD therapy. The biocompatible MNPs exclusively accumulate in the RPE layer and exert excellent antioxidative effects, which lead to alleviate pathological 
damages of AMD. Our study takes advantage of the antioxidant properties inherent to melanin as a safe and effective therapeutic strategy to combat oxidative stress and may lead to a novel treatment targeted to preserve the RPE and photoreceptors in AMD.

\section{Experiment Section}

\subsection{Synthesis of melanin nanoparticles}

MNPs were synthesized according to a previously published method with minor modifications. In brief, dopamine hydrochloride (180 mg) was dissolved in $90 \mathrm{~mL}$ deionized water and then 780 $\mu \mathrm{L} \mathrm{NaOH}(1 \mathrm{~N})$ solution was added, followed by gentle stirring at $50{ }^{\circ} \mathrm{C}$. The mixture color instantly turned to pale yellow and gradually changed to dark brown. After reacting for $5 \mathrm{~h}$, MNPs were purified with deionized water three times by centrifugation (Thermo Scientific ${ }^{\mathrm{TM}}$ Sorvall $^{\mathrm{TM}}$ ST 16R centrifuge, USA) at 13,000 rpm for $15 \mathrm{~min}$. The MNPs were finally redispersed in deionized water $(5 \mathrm{~mL})$ and stored at room temperature.

\subsection{Surface modification of MNPs}

$10 \mathrm{~mL}$ of Bare-MNPs solution ( $1 \mathrm{mg} \mathrm{mL}^{-1}$ ) were adjusted to be $\mathrm{pH} 9-10$ by adding $\mathrm{NH}_{4} \mathrm{OH}$ solution (28 wt\%, Sigma-Aldrich) and then $50 \mathrm{mg}$ of mPEG-SH (average Mn 2,000, SigmaAldrich) was added into the mixed solution under continuous stirring for $12 \mathrm{~h}$. After the reaction was completed, the MNPs were collected by centrifugation at 13,000 rpm for 15 min, washing the pellet with deionized water three times. Characterization of MNPs was carried out by TEM, DLS, zeta-potential and FT-IR analysis as described in the Supporting Information.

\subsection{Colloidal stability of MNPs}

MNPs were prepared by centrifuging $\left(13,000 \mathrm{rpm}, 20^{\circ} \mathrm{C}, 15 \mathrm{~min}\right)$ and redispersing the pellet in different solutions including monkey vitreous, cell cultured media, saline, and deionized water. The colloidal stability of MNPs was measured by DLS analysis at intervals of a week.

\subsection{Radical scavenging assay}

To measure free radical-scavenging capacity of MNPs, DPPH scavenging method was carried out with slight modifications. We prepared a fresh stock solution of ethanol containing DPPH (0.1 $\mathrm{mM}$ ) solution and added a certain amount of MNPs with the final concentration at $0,5,10,20,30$, 50, and $100 \mathrm{ug} \mathrm{mL} \mathrm{mL}^{-1}$. All solutions were placed in a dark condition for 20 min and the absorbance 
was monitored at $516 \mathrm{~nm}$ by a spectrophotometer (Molecular Devices Spectramax M5) every 5 min for 30 min. DPPH solutions at the same concentration containing no tested samples were used as control. The DPPH scavenging effect was calculated by following equation:

DPPH scavenging effect $(\%)=[1-(\mathrm{A} i-\mathrm{A} j) / \mathrm{Ac}] \mathrm{X} 100 \%$

Where Ac is the absorbance of control (without tested samples), Ai is the absorbance of samples (with MNPs), and Aj is the absorbance of the samples without DPPH solution (only MNPs). Samples were analyzed in triplicate.

\subsection{Cell culture}

ARPE19 cell line was used for in vitro studies. The cells were cultured in T-flask containing DMEM/F-12+GlutaMAX-I (Gibco, Cat. No. 10565018) supplemented with $10 \%$ fetal bovine serum (FBS, Sigma, and Cat. No. 151400122) and 1 \% antibiotic (penicillin/streptomycin) solution and incubated in a humidified incubator (Thermo Fisher Scientific, USA) at $37{ }^{\circ} \mathrm{C}$ under $5 \% \mathrm{CO}_{2}$ atmosphere.

\subsection{Cytotoxicity of MNPs in ARPE19 cells}

A WST-1 assay was carried out to measure cell viability, following the manufacturer's protocol. For the experiment, ARPE19 cells were seeded in 96 well plates at a density of $8 \times 10^{3}$ cells per well $(n=4)$ and allowed to grow at a humidified cell culture incubator for $24 \mathrm{~h}$. The cells were then exposed to $200 \mu \mathrm{L}$ of fresh media containing different concentrations $(0,5,10,20,30,50$ and 100 $\mu \mathrm{g} \mathrm{mL}^{-1}$ ) of MNPs. The plates were continuously incubated for different time periods (1 - 2 days). At the end of the incubation period, the WST-1 reagent (Abcame, ab65473, USA) was added to each well and additionally incubated at $27^{\circ} \mathrm{C}$ for $2 \mathrm{~h}$. Absorbance of cell culture medium was measured using a spectrophotometer at a wavelength of $450 \mathrm{~nm}$ with a background subtraction at $630 \mathrm{~nm}$. The absorbance values of control (untreated cells) were used as a reference value for determining the cell viability (\%).

\subsection{Intracellular ROS measurement}

Based on the demonstrated effectiveness of MNPs as robust ROS scavengers, the intracellular ROS level was detected by using Reactive Oxygen Species Assay Kit (DCFH-DA, Invitrogen ${ }^{\mathrm{TM}}$ ). This method uses a fluorogenic probe as a sensitive marker of cellular oxidation process and 
indirectly determines intracellular ROS level in cells. Once the DCFH-DA penetrates through the cell membrane, it is readily deacetylated to DCFH, which is further oxidized by intracellular ROS to a highly fluorescent compound (fluorescent 2',7'-dichlorofluorescein (DCF)) in the cells. ARPE 19 cells were seeded into 96-well plates at a density of $8 \times 10^{3}$ cells per well ( $\mathrm{n}=4$ ) for 24 $\mathrm{h}$, followed by the treatment of MNPs in different concentrations $\left(0,2,4,6,8\right.$, and $\left.10 \mu \mathrm{gL}^{-1}\right)$. After $24 \mathrm{~h}$ of incubation with increasing concentration, the cells were washed with serum-free media and loaded $100 \mu \mathrm{L}$ fresh media containing $50 \mu \mathrm{M}$ DCFH-DA solution to each well with remaining untreated cells as control. The plate was then incubated at $37^{\circ} \mathrm{C}$ for $60 \mathrm{~min}$. The cells were washed with $1 \mathrm{X}$ DPBS and treated with $100 \mu \mathrm{L}$ fresh media containing $\mathrm{H}_{2} \mathrm{O}_{2}$ (final concentration: $0.575 \mathrm{mM}$ ) to each well, and then incubated for $2 \mathrm{~h}$ at $37^{\circ} \mathrm{C}$. Only DCFH-DA treated and untreated cells were used as controls. The DCF fluorescent intensity was measured by a spectrophotometer (Molecular Devices Spectramax M5) with excitation and emission wavelengths at 485 and $530 \mathrm{~nm}$, respectively. To reliably measure levels of ROS in live ARPE19 cells, $5 \mu \mathrm{M}$ (final concentration) of a fluorogenic probe (MitoSoX, Thermo Fisher Scientific Invitrogen $^{\mathrm{TM}}$, USA) was added to each well after MNPs incubation and $\mathrm{H}_{2} \mathrm{O}_{2}$ treatment. The cells were then washed twice with 1 X DPBS and measured intracellular ROS level in the live ARPE19 cells with excitation and emission at $510 \mathrm{~nm}$ and $580 \mathrm{~nm}$, respectively.

\subsection{Embedding and thin sectioning with ultramicrotome}

To explore a behavior of MNPs in ARPE19 cells, the cells were incubated in 6 well plate at a density of $1.5 \times 10^{5}$ cells per well and exposed to $2 \mathrm{~mL}$ of fresh media containing of MNPs (100 $\mu \mathrm{g} \mathrm{mL}{ }^{-1}$ ). After $24 \mathrm{~h}$, ARPE19 Cells (with non-treatment and/or MNPs-treatment) were fixed in 2.5\% glutaraldehyde (25\%, Sigma-Aldrich) in sodium phosphate buffer (0.15M, pH 7.4$)$ for $1 \mathrm{~h}$ at room temperature and stored at $4^{\circ} \mathrm{C}$ until next processed. After rinsing three times with sodium phosphate buffer (0.15M, pH 7.4), the specimens were post-fixed in 1\% osmium tetroxide (microscopy grade, Sigma-Aldrich) for $1 \mathrm{~h}$ at room temperature. After washing with sodium phosphate buffer (0.15M, pH 7.4), the specimens were dehydrated (30\%, 50\%, 75\%, 100\%, 100\%, 10 minutes each) with increasing concentrations of ethanol and embedded in Polybed 812 epoxy resin (Polysciences, Inc.) to make plastic blocks. The small specimen blocks were sectioned to 100 nm thickness by a diamond knife using a Cryo-ultramicrotome (Leica EM UC7, CHANL-UNC). The ultrathin sections were then collected on 200 mesh copper grids and stained with $4 \%$ aqueous 
uranyl acetate (98\% EM grade, Polysciences, Inc.) for 5 min. The visualization was aided using a Thermo Scientific ${ }^{\mathrm{TM}}$ Talos F200X transmission electron microscope operating at 200kV, and digital images were acquired using a Ceta camera on Velox-acquisition software.

\subsection{Animals}

We used postnatal age 6 - 8 weeks C57BL/6J mice (Jackson Laboratory, ME, USA) for all in vivo experiments. All animal treatments or procedures were carried out and maintained in accordance with National Institutes Health animal care guidelines as approved by the UNC Institutional Animal Care and Use Committee (IACUC).

\subsection{Laser-induced CNV mouse model}

To obtain a laser-induced CNV mice model, we followed the standard protocol. In brief, adult 68 weeks C57BL/6 mice (20 - 25 g) were randomly divided into the three groups; saline-treated, aflibercept-treated, and MNPs-treated groups (20 mice per group) and the animals were anesthetized by intraperitoneal administration of a mixture of ketamine (85 mg kg-1) and xylazine (14 mg kg-1) (Butler Schein Animal Health, Dublin OH, USA). 1\% tropicamide (Bausch \& Lomb Inc., Tampa, FL, USA) was then dropped topically to the pupils for dilation, and GenTeal lubricant gel (Alcon) was covered on the eye to avoid any eye dehydration and loss of ocular transparency. Three lesions around the optic nerve were then implemented using laser photocoagulation (532 $\mathrm{nm}, 440 \mathrm{~mW}, 80 \mathrm{~ms}$ ), and the injury on the Br membrane was confirmed by the appearance of an air bubble sign using a Micron III retinal image-guided laser system (Phoenix Research Laboratories, CA, USA). To observe the detailed morphological changes in mouse eyes, optic coherence tomography (OCT) and fundus imaging test were carried out as described in the Supporting Information 1.3. The IVT injections were carried out after two days of photocoagulation following earlier protocols. Briefly, a hole was first made on sclera of each eye using a sterile 30-gauge (G) needle. $1 \mu \mathrm{L}$ of MNPs $\left(1 \mu \mathrm{g} \mu \mathrm{L}^{-1}\right)$, aflibercept (40 $\mu \mathrm{g} \mu \mathrm{L}^{-1}$ ), and saline solution were slowly injected into the vitreous cavity using a 35 gauge needle attached to a $10 \mu \mathrm{L}$ nanofil syringe through the punctured hole of the scleral surface, and the position of the needles within the eye was visualized using a microscope (Carl Zeiss Surgical, Incorporated, Thornwood, NY, USA). In case of in vivo trafficking study, $1 \mu \mathrm{L}$ of MNPs $\left(10 \mu \mathrm{g} \mu \mathrm{L}^{-1}\right)$ and saline solution were slowly injected into the vitreous cavity of normal mouse (Non-CNV model), RPE/Choroid/Scleral tissues at 3, 15, 30, and 90 days post-injection were embedded following 
procedures as described above. The needle was gently removed from the eye and triple antibiotic ointment (Equate, Wal-Mart, Bentonville, AR, USA) was dropped on the eye surface to avoid any infections. After all surgeries, the mice were kept warm for 20 min and sacrificed to collect tissues at the end point of our study.

\subsection{Flat mounts and histology scan}

After performing the last OCT and FFA imaging, the mice were euthanized for preparing laserinduced CNV RPE/choroid/scleral flat mounts as previously described. ${ }^{17-18}$ Briefly, the eyes of the mice were enucleated and immediately fixed with $4 \%$ paraformaldehyde for $1 \mathrm{~h}$ at room temperature. After washing three times with PBS, the RPE/choroid/scleral tissues were isolated and stained with Alexa Fluor 488-conjugated isolectin-IB4 (10 $\mu \mathrm{g} \mathrm{mL}^{-1}$, Invitrogen ${ }^{\mathrm{TM}}$ ) overnight. The IB4-labeled tissues were fully washed with PBS three times for $15 \mathrm{~min}$ each and then prepared for 4 or 5 radial incisions. The tissues were flattened and mounted with the vitreous side up onto the slide. The images were visualized by Axiocam MR 5 camera on an Axio Observer.D1 inverted microscope (Carl Zeiss, Norway). For histologic analysis, the collected eyes were fixed with 4 \% paraformaldehyde and embedded in paraffin, followed by sectioning and H\&E staining at the QuantCenter 3DHISTECH, USA.

\subsection{Statistics}

All studies were triplicated for each group and the results were reported as the mean $\pm \operatorname{standard}$ deviation (S.D.). GraphPad Prism 9.0 software (La Jolla, CA, USA) was used to analyze all data also for statistical and kinetic analysis. Multiple group comparisons were examined by one-way analysis of variance (ANOVA) to determine the statistical differences; differences were considered as statistically significant at a p-value lower than $0.05(\mathrm{P}<0.05)$.

\section{Acknowledgements}

The authors thank Amar Kumbhar (The Chapel Hill Analytical and Nanofabrication Laboratory, CHANL, UNC-CH) and Victoria J Madden (The Microscopy Services Laboratory, MSL, UNC$\mathrm{CH}$ ) for their kind help with the transmission electric microscopy imaging. The authors thank Ahra Cho for her critical reading of the manuscript. This work was supported in part by the Bright Focus Foundation (M2019063, Z.H.) and the Edward N. \& Della L. Thome Memorial Foundation (138289, Z.H.). 


\section{Figure legends}

a)

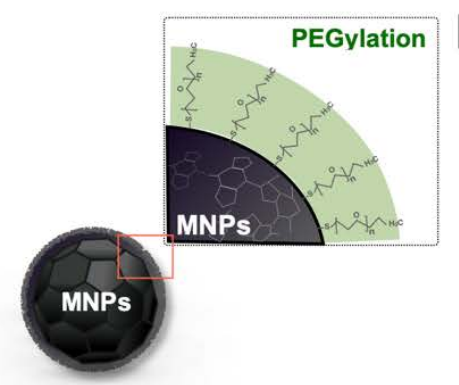

c)

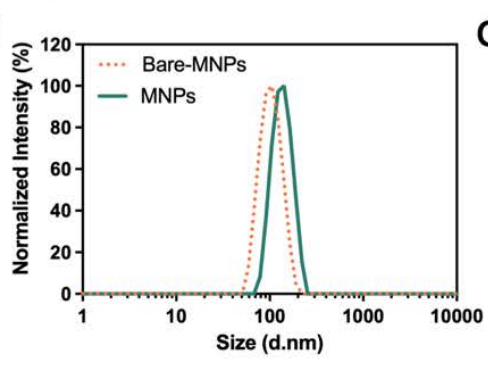

e)

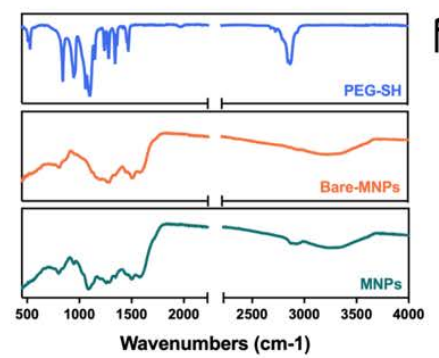

b)
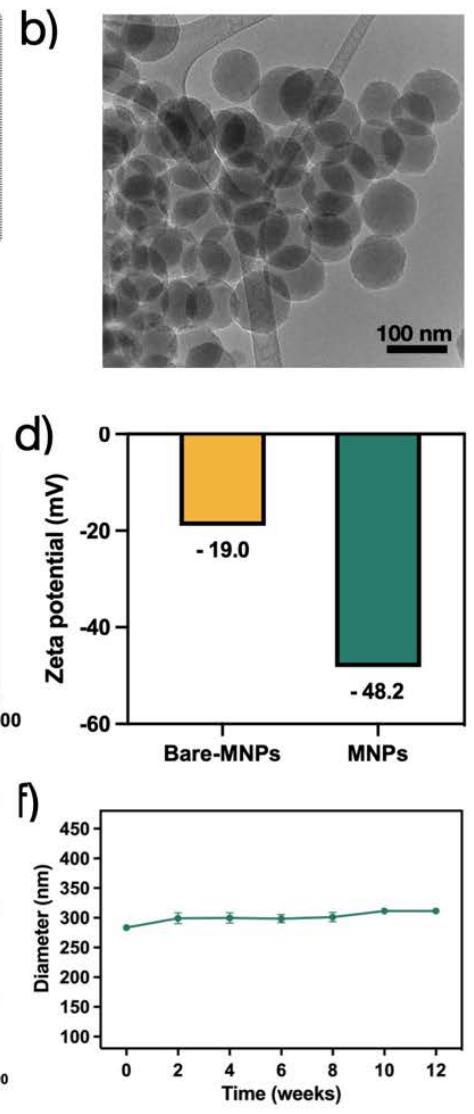

Figure 1. Characterization of nanoparticles. a) Schematic illustration of PEGylated-synthetic melanin nanoparticles (MNPs) structure. b) A transmission electron microscopy (TEM) image shows MNPs with spherical morphology and uniform size $(\sim 80 \mathrm{~nm})$. c) Dynamic light scattering (DLS) analysis measures the hydrodynamic size of Bare-MNPs and MNPs to determine the size distribution of nanoparticles and relative monodispersity. d) Zeta potential of Bare-MNPs and MNPs. The results exhibited that the average surface charge was $-19.0 \mathrm{mV}$ and $-48.2 \mathrm{mV}$, respectively. e) FT-IR spectra of mPEG-SH (2 KDa), Bare-MNPs, and MNPs. The characteristic spectral peaks of PEG appeared around $2880 \mathrm{~cm}^{-1}$ (alkyl C-H stretching) and $1110 \mathrm{~cm}^{-1}$ (C-0-C stretching). F) Colloidal stability of MNPS was measured in monkey vitreous for $\sim 12$ weeks. 

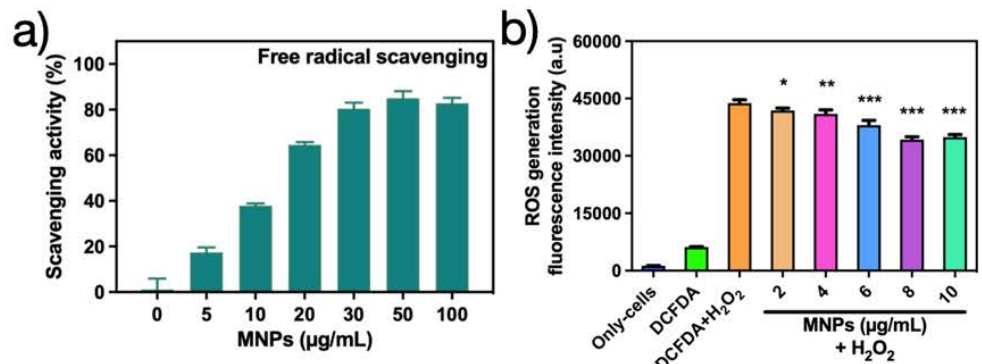

d)

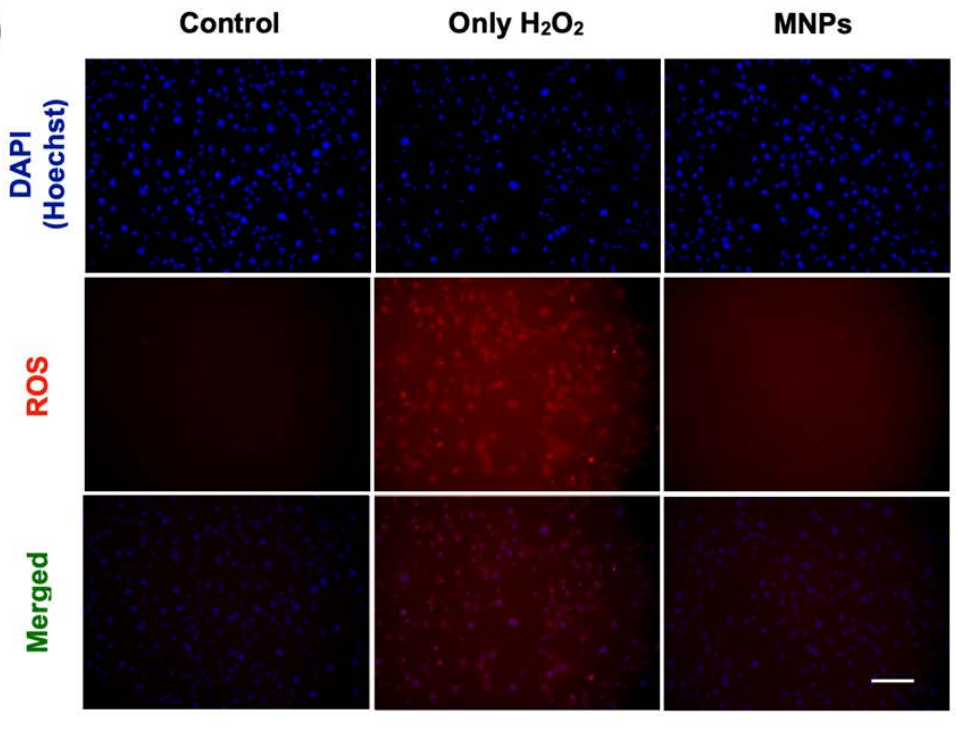

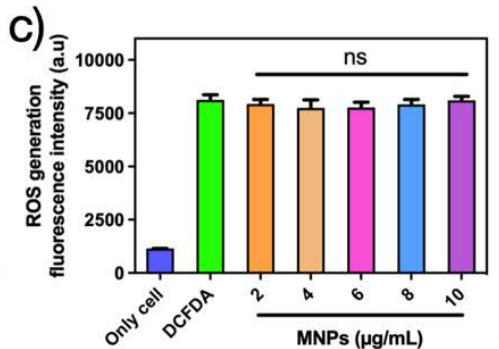

e)
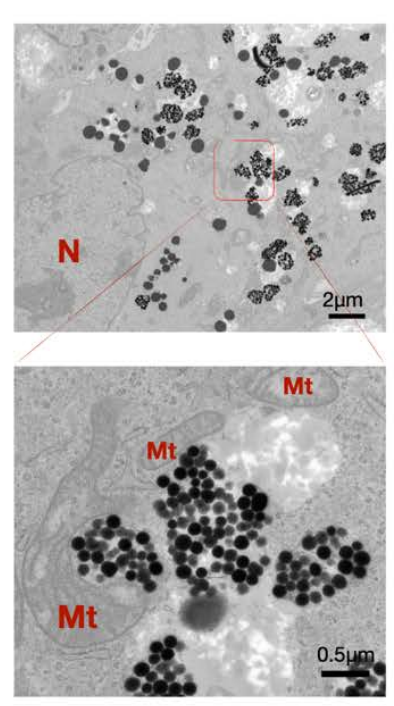

Figure 2. ROS-scavenging activity of MNPS in ARPE19 cells. a) The capacity of MNPs to scavenge free radicals in APRE19 cells, b) Intracellular ROS scavenging activity of MNPS at different concentrations in $\mathrm{H}_{2} \mathrm{O}_{2}$-treated $(0.575 \mathrm{mM})$ ARPE 19 cells using DCFH-DA $(50 \mu \mathrm{M})$ and. c) Intracellular ROS measurement following the same method as described above with no $\mathrm{H}_{2} \mathrm{O}_{2}$ treatment. d) Representative fluorescence microscopy images showing the ROS staining of ARPE19 cells under different treatments. Scale bar: $100 \mu \mathrm{m}$. e) Bio-TEM images showed exclusively located MNPs in cytosol of ARPE 19 cell but not in the nucleus and mitochondria (N; nuclear, Mt; mitochondria). ns, nonsignificant; ${ }^{*} \mathrm{p}<0.05$, ${ }^{* *} \mathrm{P}<0.01$, and $* * * \mathrm{P}<0.001$ versus blank group, $n=4$. All data are presented as the mean \pm S.D. 

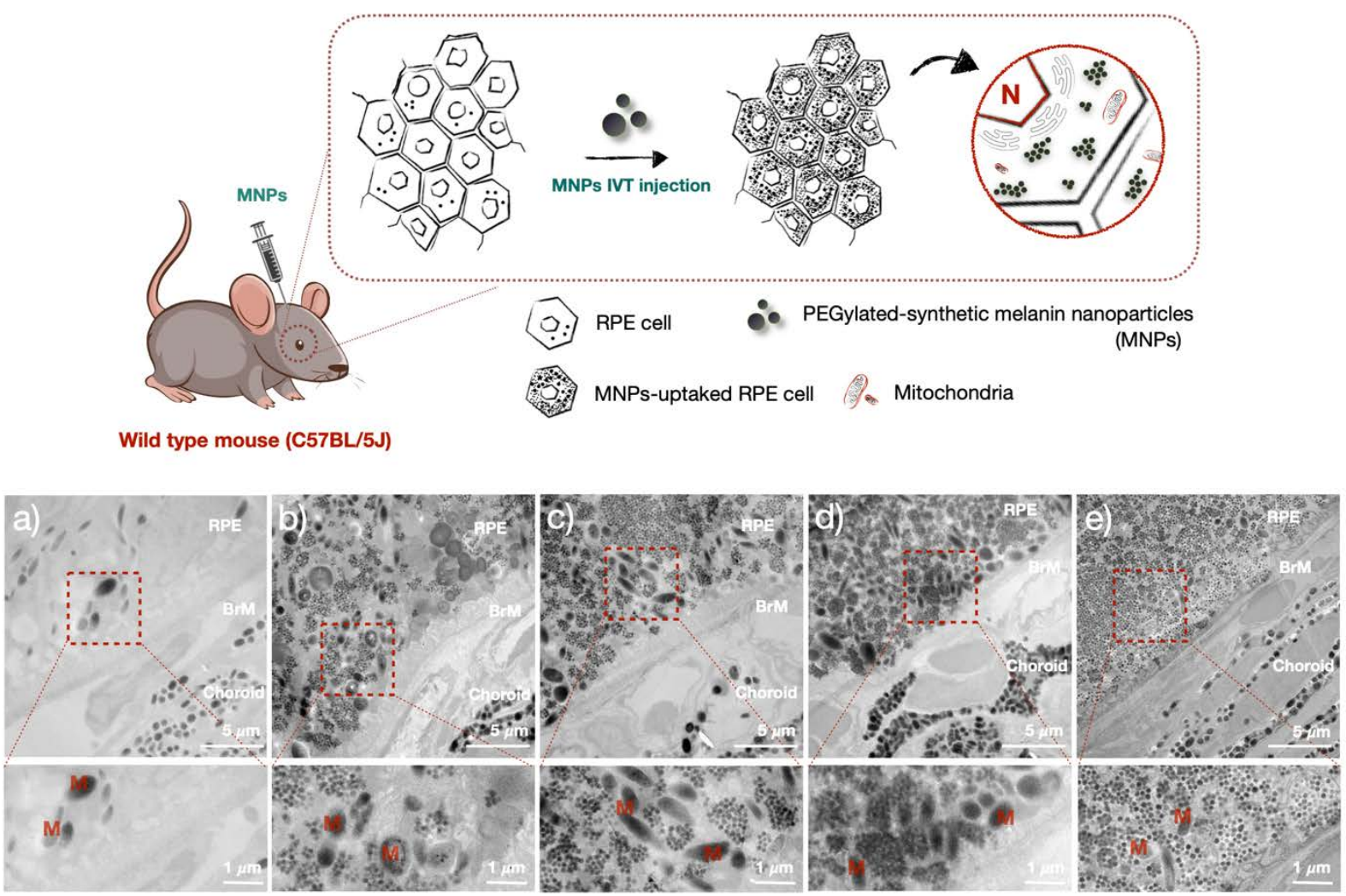

Figure 3. Comparison of the intracellular trafficking of MNPs in ocular tissues a) before IVT administration and after administration at b) 3 days, c) 15 days, d) 30 days, and e) 90 days, respectively. The Bio-TEM images show the presence of MNPs in the RPE layer from 3 days after exposure and reveal a large number of trapped MNPs inside RPE layer. Higher magnification of the tissues where nanoparticles are present, indicated by bottom images. BrM: bruch’s membrane, M: melanosome. 


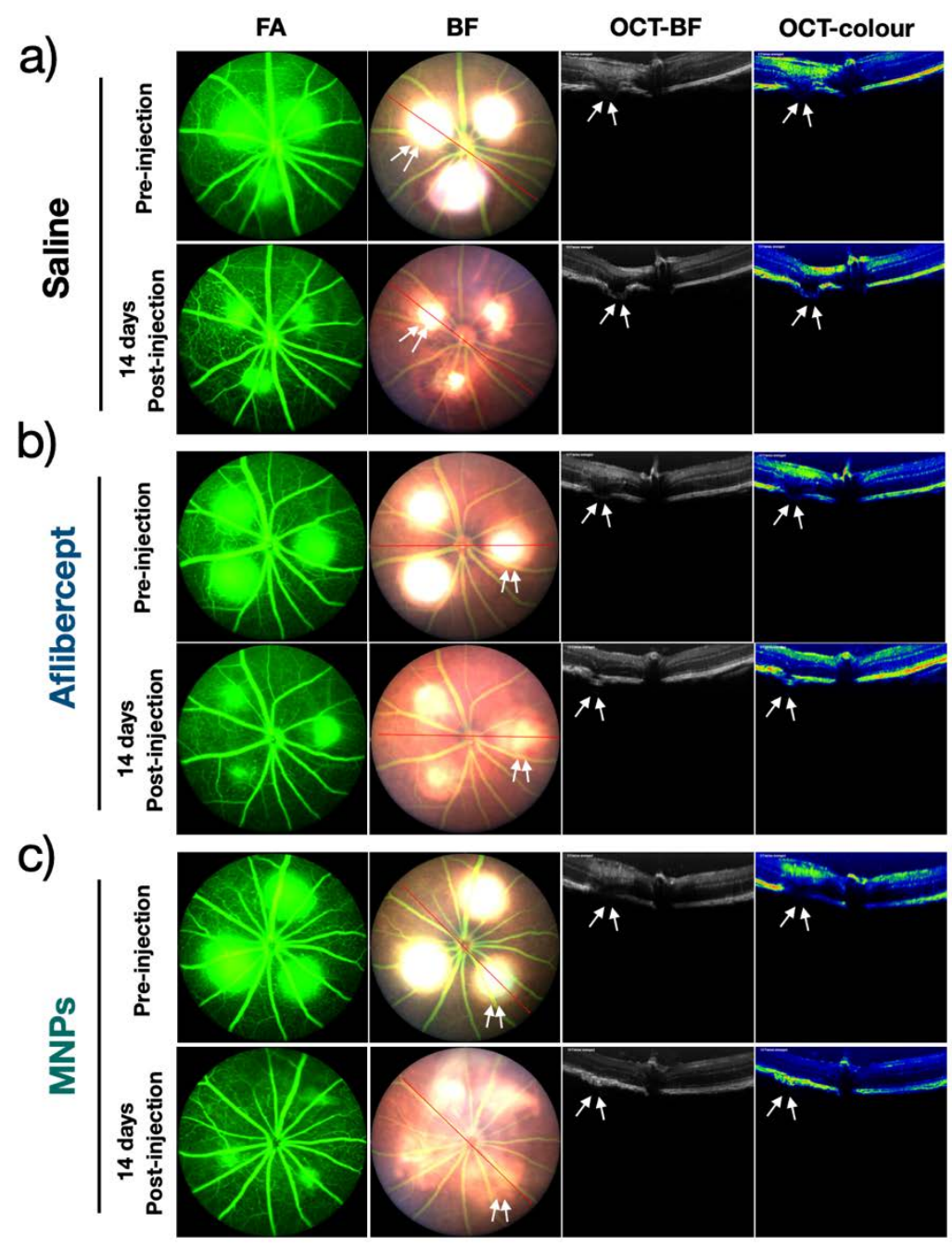

Figure 4. Fundus and OCT photographs analyses of choroidal neovascularization in a laserinduced CNV mouse model after IVT injection. a) Representative of fundus/OCT photographs in saline-treated eyes, which show just before injection (top panel) and after 14 days of laser injuries (bottom panel). b) Representative of fundus/OCT photographs in aflibercept-treated eyes, which show just before injection (top panel) and after 14 days of laser injuries (bottom panel). c) Representative of fundus/OCT photographs for MNPs-treated eyes, which show just before injection (top panel) and after 14 days of laser injuries (bottom panel). White arrows indicate the areas of laser damage. Fluorescein angiography: FA, and bright field: BF. 
a)

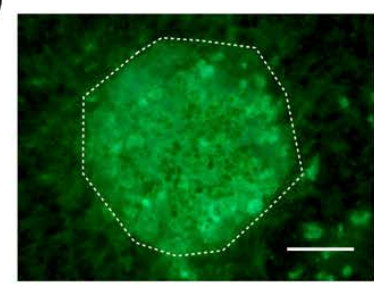

Saline

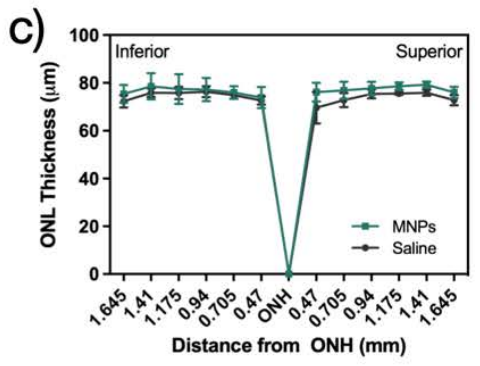

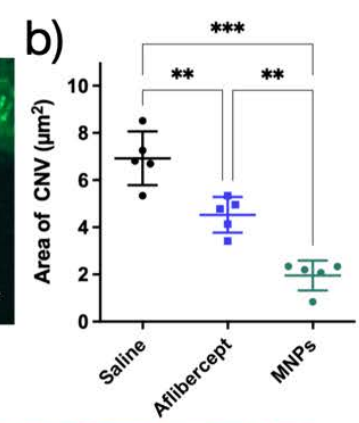

d)

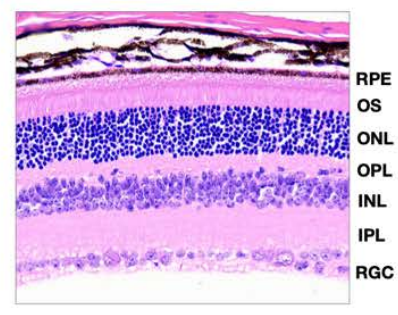

e)

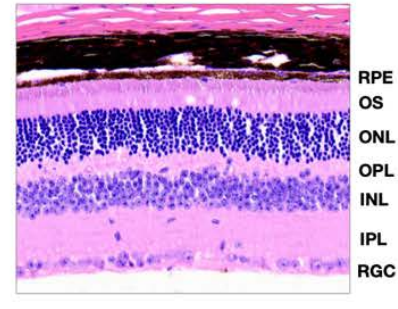

Figure 5. a) Representative images of RPE/choroid/scleral flat mounts from saline- and aflibercept-, and MNPs-treated eyes. Scale bar $=100 \mu \mathrm{m}$. b) Quantification of laser-induced CNV areas $\left(\mu \mathrm{m}^{2}\right)$. Each dot represents one CNV lesion site. $\mathrm{n}=5$ lesions/group. Data are presented as mean \pm SEM. one-way ANOVA followed by Tukey's post hoc multiple comparison test, $* * \mathrm{P}<0.01$, and $* * * \mathrm{P}$ $<0.001$. c) Quantification of outer nuclear layer (ONL) thickness. There are no significant changes in ONL thickness between saline- and MNPs-treated groups. Data are represented as mean \pm S.D. Histological analysis of retinas in d) saline- and e) MNPs-treated groups. 


\section{References}

1. Handa, J. T.; Bowes Rickman, C.; Dick, A. D.; Gorin, M. B.; Miller, J. W.; Toth, C. A.; Ueffing, M.; Zarbin, M.; Farrer, L. A., A systems biology approach towards understanding and treating nonneovascular age-related macular degeneration. Nature Communications 2019, 10 (1), 3347.

2. Wong, W. L.; Su, X.; Li, X.; Cheung, C. M.; Klein, R.; Cheng, C. Y.; Wong, T. Y., Global prevalence of age-related macular degeneration and disease burden projection for 2020 and 2040: a systematic review and meta-analysis. The Lancet. Global health 2014, 2 (2), e106-16.

3. Wright, C. B.; Ambati, J., Dry Age-Related Macular Degeneration Pharmacology. Handb Exp Pharmacol 2017, 242, 321-336.

4. Beatty, S.; Koh, H.-H.; Phil, M.; Henson, D.; Boulton, M., The Role of Oxidative Stress in the Pathogenesis of Age-Related Macular Degeneration. Survey of Ophthalmology 2000, 45 (2), 115-134.

5. Ozawa, Y., Oxidative stress in the light-exposed retina and its implication in age-related macular degeneration. Redox Biology 2020, 37, 101779.

6. Nita, M.; Grzybowski, A., The Role of the Reactive Oxygen Species and Oxidative Stress in the Pathomechanism of the Age-Related Ocular Diseases and Other Pathologies of the Anterior and Posterior Eye Segments in Adults. Oxid Med Cell Longev 2016, 2016, 3164734-3164734.

7. Holz, F. G.; Schmitz-Valckenberg, S.; Fleckenstein, M., Recent developments in the treatment of age-related macular degeneration. J Clin Invest 2014, 124 (4), 1430-1438.

8. Ambati, J.; Atkinson, J. P.; Gelfand, B. D., Immunology of age-related macular degeneration. Nat Rev Immunol 2013, 13 (6), 438-451.

9. Roggia, M. F.; Imai, H.; Shiraya, T.; Noda, Y.; Ueta, T., Protective Role of Glutathione Peroxidase 4 in Laser-Induced Choroidal Neovascularization in Mice. PLOS ONE 2014, 9 (6), e98864.

10. Dogru, M.; Kojima, T.; Simsek, C.; Tsubota, K., Potential Role of Oxidative Stress in Ocular Surface Inflammation and Dry Eye Disease. Investigative Ophthalmology \& Visual Science 2018, 59 (14), DES163DES168.

11. Pinazo-Durán, M. D.; Gallego-Pinazo, R.; García-Medina, J. J.; Zanón-Moreno, V.; Nucci, C.; DolzMarco, R.; Martínez-Castillo, S.; Galbis-Estrada, C.; Marco-Ramírez, C.; López-Gálvez, M. I.; Galarreta, D. J.; Díaz-Llópis, M., Oxidative stress and its downstream signaling in aging eyes. Clinical interventions in aging 2014, 9, 637-52.

12. Taubitz, T.; Tschulakow, A. V.; Tikhonovich, M.; Illing, B.; Fang, Y.; Biesemeier, A.; JulienSchraermeyer, S.; Schraermeyer, U., Ultrastructural alterations in the retinal pigment epithelium and photoreceptors of a Stargardt patient and three Stargardt mouse models: indication for the central role of RPE melanin in oxidative stress. PeerJ 2018, 6, e5215.

13. Schraermeyer, U.; Kopitz, J.; Peters, S.; Henke-Fahle, S.; Blitgen-Heinecke, P.; Kokkinou, D.; Schwarz, T.; Bartz-Schmidt, K.-U., Tyrosinase biosynthesis in adult mammalian retinal pigment epithelial cells. Experimental Eye Research 2006, 83 (2), 315-321.

14. Lapierre-Landry, M.; Carroll, J.; Skala, M. C., Imaging retinal melanin: a review of current technologies. Journal of Biological Engineering 2018, 12 (1), 29.

15. Gu, X.; Neric, N. J.; Crabb, J. S.; Crabb, J. W.; Bhattacharya, S. K.; Rayborn, M. E.; Hollyfield, J. G.; Bonilha, V. L., Age-related changes in the retinal pigment epithelium (RPE). PloS one 2012, 7 (6), e38673e38673.

16. Sarna, T.; Burke, J. M.; Korytowski, W.; Różanowska, M.; Skumatz, C. M. B.; Zaręba, A.; Zaręba, M., Loss of melanin from human RPE with aging: possible role of melanin photooxidation. Experimental Eye Research 2003, 76 (1), 89-98.

17. Wang, K.; Zheng, M.; Lester, K. L.; Han, Z., Light-induced Nrf2-/- mice as atrophic age-related macular degeneration model and treatment with nanoceria laden injectable hydrogel. Scientific Reports 2019, 9 (1), 14573. 
18. Mitra, R. N.; Gao, R.; Zheng, M.; Wu, M. J.; Voinov, M. A.; Smirnov, A. I.; Smirnova, T. I.; Wang, K.; Chavala, S.; Han, Z., Glycol Chitosan Engineered Autoregenerative Antioxidant Significantly Attenuates Pathological Damages in Models of Age-Related Macular Degeneration. ACS nano 2017, 11 (5), 4669-4685.

19. Liu, H.; Yang, Y.; Liu, Y.; Pan, J.; Wang, J.; Man, F.; Zhang, W.; Liu, G., Melanin-Like Nanomaterials for Advanced Biomedical Applications: A Versatile Platform with Extraordinary Promise. Advanced Science 2020, 7 (7), 1903129.

20. Ju, K.-Y.; Lee, Y.; Lee, S.; Park, S. B.; Lee, J.-K., Bioinspired Polymerization of Dopamine to Generate Melanin-Like Nanoparticles Having an Excellent Free-Radical-Scavenging Property.

Biomacromolecules 2011, 12 (3), 625-632.

21. Lee, H.; Dellatore, S. M.; Miller, W. M.; Messersmith, P. B., Mussel-inspired surface chemistry for multifunctional coatings. Science (New York, N.Y.) 2007, 318 (5849), 426-30.

22. Kedare, S. B.; Singh, R. P., Genesis and development of DPPH method of antioxidant assay. J Food Sci Technol 2011, 48 (4), 412-422.

23. Kim, E.; Kang, M.; Tschirhart, T.; Malo, M.; Dadachova, E.; Cao, G.; Yin, J.-J.; Bentley, W. E.; Wang, Z.; Payne, G. F., Spectroelectrochemical Reverse Engineering DemonstratesThat Melanin's Redox and Radical Scavenging Activities Are Linked. Biomacromolecules 2017, 18 (12), 4084-4098.

24. Zorov, D. B.; Juhaszova, M.; Sollott, S. J., Mitochondrial reactive oxygen species (ROS) and ROSinduced ROS release. Physiological reviews 2014, 94 (3), 909-50.

25. Lujan, B. J.; Roorda, A.; Croskrey, J. A.; Dubis, A. M.; Cooper, R. F.; Bayabo, J.-K.; Duncan, J. L.; Antony, B. J.; Carroll, J., DIRECTIONAL OPTICAL COHERENCE TOMOGRAPHY PROVIDES ACCURATE OUTER NUCLEAR LAYER AND HENLE FIBER LAYER MEASUREMENTS. Retina 2015, 35 (8), 1511-1520.

26. Sarna, T., New trends in photobiology: Properties and function of the ocular melanin - a photobiophysical view. Journal of Photochemistry and Photobiology B: Biology 1992, 12 (3), 215-258. 27. Weiter, J. J.; Delori, F. C.; Wing, G. L.; Fitch, K. A., Retinal pigment epithelial lipofuscin and melanin and choroidal melanin in human eyes. Invest Ophthalmol Vis Sci 1986, 27 (2), 145-52.

28. Wu, L.; Nagasaki, T.; Sparrow, J. R., Photoreceptor cell degeneration in Abcr (-/-) mice. Advances in experimental medicine and biology 2010, 664, 533-9.

29. LaVail, M. M.; Sidman, R. L.; Gerhardt, C. O., Congenic strains of RCS rats with inherited retinal dystrophy. The Journal of heredity 1975, 66 (4), 242-4.

30. Zhang, J.; Bai, Y.; Huang, L.; Qi, Y.; Zhang, Q.; Li, S.; Wu, Y.; Li, X., Protective effect of autophagy on human retinal pigment epithelial cells against lipofuscin fluorophore A2E: implications for age-related macular degeneration. Cell Death \& Disease 2015, 6 (11), e1972-e1972.

31. Di Mauro, E.; Xu, R.; Soliveri, G.; Santato, C., Natural melanin pigments and their interfaces with metal ions and oxides: emerging concepts and technologies. MRS Communications 2017, 7 (2), 141-151.

32. Foroozandeh, P.; Aziz, A. A., Insight into Cellular Uptake and Intracellular Trafficking of Nanoparticles. Nanoscale Res Lett 2018, 13 (1), 339-339.

33. Krohne, T. U.; Eter, N.; Holz, F. G.; Meyer, C. H., Intraocular Pharmacokinetics of Bevacizumab After a Single Intravitreal Injection in Humans. American Journal of Ophthalmology 2008, 146 (4), 508512.

34. Sousa, F.; Castro, P.; Fonte, P.; Kennedy, P. J.; Neves-Petersen, M. T.; Sarmento, B., Nanoparticles for the delivery of therapeutic antibodies: Dogma or promising strategy? Expert opinion on drug delivery 2017, 14 (10), 1163-1176. 